\title{
Un chantier naval à Hochiminh-Ville
}

A naval building site in Hochiminh-Ville

Un astillero en Hochiminh-ville

\section{Laura Bogani}

\section{OpenEdition}

\section{Journals}

Édition électronique

URL : https://journals.openedition.org/tc/295

DOI : $10.4000 /$ tc. 295

ISSN : 1952-420X

Éditeur

Éditions de l'EHESS

Édition imprimée

Date de publication : 1 janvier 2001

Pagination : 287-300

ISSN : 0248-6016

Référence électronique

Laura Bogani, « Un chantier naval à Hochiminh-Ville», Techniques \& Culture [En ligne], 35-36 | 2001, mis en ligne le 07 septembre 2012, consulté le 29 septembre 2022. URL : http://journals.openedition.org/ tc/295; DOI : https://doi.org/10.4000/tc.295

Ce document a été généré automatiquement le 29 septembre 2022

Tous droits réservés 


\title{
Un chantier naval à Hochiminh- Ville
}

\author{
A naval building site in Hochiminh-Ville \\ Un astillero en Hochiminh-ville
}

Laura Bogani

1 Situé dans le sud de la ville, le quartier de Nhà Bè est depuis très longtemps le lieu privilégié des constructeurs navals; il est bien connu des pirates et des boat-people. C'est aussi un carrefour important entre les voies de navigation maritimes et fluviales. De la mer, on y accède par la baie de Vung Tao. On navigue sur le bras Cua Soai Rap puis le long de la rivière Sông Nhà Bè ; et de l'arroyo ${ }^{2}$ Rach Ông, on débouche dans l'arroyo chinois qui traverse Hochiminh-Ville, où sont installés les dépôts de riz et les marchés de gros. Cette voie n'est praticable par les grands tonnages qu'à marée haute. Le marnage $\mathrm{y}$ varie de un à trois mètres. Ce quartier abrite le chantier naval Đai Thắng, entres autres, très fréquenté par les bateaux nécessitant réparations ou carénage.

2 Parmi les bateaux en bois qui naviguent dans le Delta du Mékong, trois types sont intéressants quant à leur construction. Ils diffèrent par leur forme, leur décoration et leur provenance : le ghe cân đuôc, le ghe chài et le ghe cà vom.

3 Le ghe cân đúôc est un bateau fluvial. Son ventre arrondi est formé par une cale utilisée pour le transport de marchandises telles que le paddy, le sable, les briques, le ciment et le bois. Ses lignes d'eaux se terminent dans une étrave élancée qui ne permet pas l'accès direct à la cale pat l'avant du bateau. En revanche, cet élément évite que la vague d'étrave, due à la vitesse, ne vienne envahir le bateau. Les Vietnamiens le définissent ainsi : «le nez rouge pointu », mũi don màu đo. Comme beaucoup de bateaux en Asie du Sud-Est, il a des yeux peints de chaque côté de l'étrave (fig. 1). Ce type de bateau existe en deux versions : à cale ouverte ou à cale fermée, selon l'activité de son propriétaire. Le gouvernail compensé, tâm lái thắng bang, est généralement coulissant, pour permettre de passer sur les bancs de sable, les hauts fonds et les arroyos trop peu profonds. Il remonte jusqu'au toit du bateau et se prolonge le long d'une barre franche pouvant atteindre trois mètres de longueur. Le capitaine peut, de son poste de pilotage 
qui se trouve sur le toit du bateau, conduire son bateau avec une vue dégagée sur trois cent soixante degrés. Ces bateaux sont construits principalement dans la localité de Can Duoc $^{3}$, située au sud de la province de Long An, dans le Delta du Mékong.

4 Le ghe chài est l'héritier de la jonque chinoise, avec toutefois une différence fondamentale: les voiles en sont totalement absentes. Son chargement n'est jamais inférieur à cent tonnes. Les bateaux de ce type sont construits pour les commerçants chinois de Cho Lon. Ils sont souvent à cale fermée et assurent la liaison entre les provinces du Delta et le marché de gros de Cho Lon. Le gouvernail compensé et coulissant est relié à une barre à roue, plus facile à utiliser lors des manoeuvres dans les arroyos et les ports de commerce. Son étrave plate facilite le chargement et le déchargement à l'avant du bateau, «le nez rouge plat », mũi đuoc son màu đo. Les yeux sont de forme ovale ou en goutte d'eau (fig. 1). Ces bateaux sont construits principalement dans les environs de Cho Lon.

5 Le ghe cà vom diffère par sa forme des navires précédents. Il est l'héritage des pirogues monoxyles et de bateaux qui, autrefois, naviguaient dans le nord du Delta, en particulier dans les provinces de Dong Thap et de An Giang. Sa coque est symétrique, ses extrémités se prolongent par deux pièces massives. Cette fois, son étrave ressemblant à une trompe d'éléphant est appelée "nez bleu allongé », mũi đuoc kéo dài. Ce même élément se retrouve à l'arrière du bateau, lui-même très décoré et élancé. Les yeux sont petits et ronds (fig. 1); ils n'ont rien de commun avec ceux des ghe cân đuoc et des ghe chài.

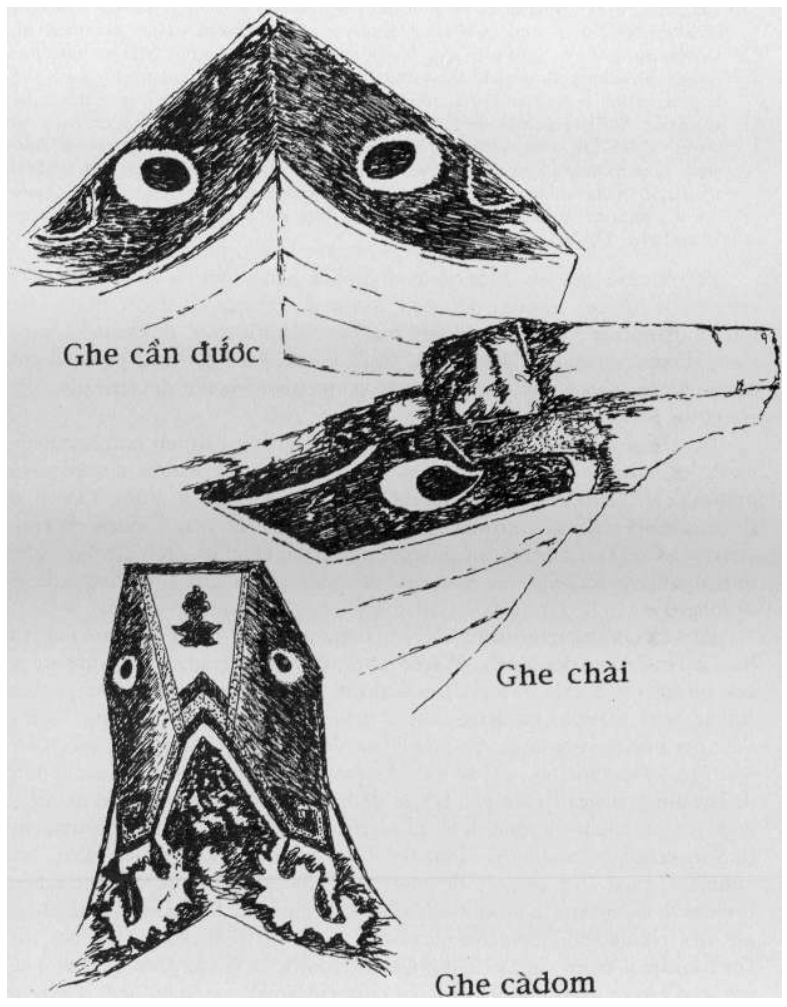

Figure 1. Les yeux

6 Ce bateau était à l'origine une pirogue, Pierre Paris en a laissé une description :

«En arrivant en Cochinchine, les Annamites ont adopté et adapté la pirogue monoxyle cambodgienne. Il est rare qu'elle soit employée rigoureusement en un seul morceau. Généralement elle est exhaussée d'un bordage maintenu par des 
membrures qui renforcent en même temps l'ensemble. Dans cet état elle sert surtout dans les canaux creusés par le propriétaire, et à rassembler la récolte au centre du domaine, près d'un grand canal navigable. Sur la gracieuse pirogue de voyage, le bordé en surélévation a été peint en couleur claire. Exhaussée davantage, la pirogue devient le bâtiment appelé Ghe cà vom. Mais alors on assiste à un phénomène remarquable: renonçant complètement au fond monoxyle, les Annamites ont construit sous le même nom une grosse jonque très élégante qui n'a conservé de la pirogue originelle que deux pièces massives aux extrémités. » (Paris $1942: 378-379)$.

7 J'ai observé que les distinctions morphologiques des bateaux selon les critères de couleur -forme des yeux, forme de l'étrave ou décoration- ne sont plus vraiment valables aujourd'hui pour définir avec précision la province d'appartenance d'un bateau. Seuls la mémoire des gens et les documents ou les photos tirés des archives permettent encore de retrouver les traces du passé.

8 J'ai choisi de privilégier le chantier Dai Thang car son emplacement révèle les relations et les échanges qui se font par voie maritime à travers la presqu'île de Can Gio, les bouches du Mékong et le port de Vung Tao. Il se situe au croisement des arroyos Rach Ông et Kinh Te, par lesquels on peut arriver à Cho Lon ainsi qu'au port commercial d'Hochiminh-Ville. Six cales sèches peuvent accueillir des bateaux de vingt-cinq à quarante-cinq mètres de long.

9 La fonction caractéristique de ce chantier d'État est la construction en bois, à l'exclusion des autres. Y sont fabriqués des bateaux de transport à cale ouverte et à cale fermée. Les bateaux à cale ouverte sont semi-pontés; seul le tiers arrière est fermé, et il sert de cabine à l'équipage. Vient s'ajouter une terrasse en porte-à-faux sur l'étambot, qui accueille la cuisine, nhà hep, et les toilettes, nhà vê sinh. Le poste de pilotage se trouve sur le toit de la cabine, il ne dépasse pas $1,5 \mathrm{~m}$ de hauteur lorsqu'il s'agit de manœuvrer avec une barre à roue. Il est plus grand et plus ouvert quand le système de gouvernail est une barre franche. Ce type de bateau est employé, notamment, pour le transport de matériaux de construction tels que sable, briques, bois, ciment. L'accès facile à la cale permet de charger et décharger très rapidement. Son gros inconvénient est un franc bord trop bas sur l'eau lorsqu'il est en charge, qui oblige à réduire la vitesse afin d'éviter une vague d'étrave trop importante pouvant entraîner son naufrage. Le problème est le même pour les péniches en Occident: la hauteur de la vague d'étrave est relative à la vitesse de la péniche. Si le franc bord est trop bas, l'eau envahit la cale, augmentant la charge et le navire coule inévitablement. Les autres bateaux ont une cale fermée par une structure architecturale en longueur, qui permet le chargement par le haut à travers des hiloires coulissants. À l'avant du bateau, on peut circuler et, par un escalier, accéder au toit où se situe la cabine de pilotage. $\mathrm{A}$ l'arrière, un autre escalier permet de descendre sur la terrasse où se trouvent le coin cuisine, les toilettes et la cabine du propriétaire. Ce type d'embarcation est très utilisé, en période de pluie, pour le transport de marchandises entre le Delta et HochiminhVille où les marchandises sont livrées aux cargos dans le port de la cité.

Le chantier Đai Thắng est entré en activité en $1957^{4}$. Dans ses cales sèches travaillent quarante personnes (trente hommes et dix femmes). Les tâches y sont clairement réparties. Les travaux de nettoyage de la coque, le calfatage, la pose du mastic et la peinture sont réservés aux femmes. Les hommes préparent le bois pour les bordés, réalisent l'assemblage, la soudure, et s'occupent de la ferronnerie et de la mécanique. Les horaires de travail des femmes et des hommes sont décalés afin d'éviter des 
interférences gênantes. Le chantier est organisé selon une hiérarchie précise et chaque groupe de travail reçoit les ordres de son propre chef.

11 Le bureau d'étude du chantier est chargé de trouver les clients et planifie le travail pour les navires en construction ou en réparation. Le client arrive parfois avec des plans ou une idée précise du bateau qu'il souhaite faire fabriquer. Les ingénieurs du bureau étudient avec lui les modalités de la construction : ils lui montrent des photos et des maquettes de bateaux et le conseillent. Mais les ingénieurs travaillent très rarement sur plans, car la tradition orale et le gabarit priment encore.

Le plus souvent, le chef du chantier rencontre les charpentiers, thô đong tàu (ou thô môc) pour commenter le projet; il dessine sur un tableau noir les pièces maîtresses ainsi que leur emplacement. Une fois définis la longueur, la largeur, le tirant d'eau, les charpentiers établissent une aire de travail sur laquelle sera posé le fond du bateau, ou le cas échéant, sa quille (les bateaux fluviaux, par exemple, sont dépourvus de quille).

Il faut ensuite trouver le bois d'oeuvre. Au Vietnam, le sao (Hopea Odorata) est l'essence la plus fréquemment utilisée. Ce bois, facile à travailler, résiste aux insectes et se comporte très bien dans l'eau. Sa couleur, jaune clair, caractéristique lors de la coupe, devient brun clair sous l'effet du soleil et prendra avec le temps une jolie patine. Une fois coupé en forêt, le bois est transporté dans des dépôts qui font office de réserve et de lieu de vente. Il y est débité en planches pour garantir un bon séchage, puis posé sur une structure aérée afin que les planches ne se déforment pas. Le temps du séchage peut varier de quinze à trente jours. Dans les grands chantiers navals, tel Ba Son à Hochiminh-Ville, on procède au séchage du bois par étuvage - pratique assez courante aussi en Europe-, en particulier pour le bois qui sert aux aménagements intérieurs. Si le bois n'est pas correctement séché, il sera attaqué par les voraces parasites de ces latitudes et seule une fumigation permettra de les éliminer. Dans les dépôts, de grandes quantités de toutes les essences de bois sont exposées afin de permettre au client, assisté par un expert, de faire son choix. Si le client n'est pas satisfait du bois disponible, il a la possibilité de se rendre en forêt pour qu'il choisisse lui-même les arbres sur pied. Le bois est ensuite acheminé - de la forêt ou du dépôt- jusqu'au chantier naval, par camion ou par bateau.

Pour débuter la construction, la croyance populaire impose de choisir un jour faste, selon le calendrier lunaire et en rapport avec l'âge du futur propriétaire -cela afin de déterminer quel Bouddha assurera sa protection ${ }^{5}$.

15 En premier lieu, on pose la structure axiale, composée de la quille (tiếp), de l'étambot (lô lai) et de l'étrave (lô mũi $\left.i^{6}\right)$. Mais la construction traditionnelle ne prévoit pas toujours de structure axiale. Certains bateaux peuvent être construits avec cinq ou sept planches (tàu nam và bay lat), selon les dimensions désirées. On commence alors par la pose du fond, sur lequel on ajoutera par la suite la structure transversale.

\section{Le gabarit}

Le gabarit est un élément en bois qui sert a définir les angles des pièces maîtresses, telles que les membrures, công ghe (ou công gian), les varangues, đà ghe, et les allonges, bỏ chong, en rapport avec la forme finale souhaitée. Deux à cinq gabarits sont nécessaires pour réaliser l'ensemble des pièces. 
17 Les membrures et varangues, découpées à la main dans des planches de sao, sont dégrossies et équarries selon le gabarit, et dimensionnées en fonction de leur emplacement. Puis on les place dans le fond du bateau. Si leur taille ne correspond pas à l'emplacement prévu, on marque à l'aide d'un instrument pointu, vòu, la partie à enlever.

Le charpentier, à coups très précis de hache à balancier, riêù, donne à la membrure sa forme définitive, qui est posée et clouée au fond du bateau. Une fois les membrures et les varangues en place, on commencera à poser les allonges, prolongements des membrures, qui permettront d'engager le travail du bordé latéral.

19 Autrefois, le bordé, ván be, était fixé aux membrures à l'aide de chevilles tronconiques en bois, technique que l'on trouve encore employée dans la construction des bateaux le long de la côte vietnamienne ${ }^{7}$.

\section{Le cintrage du bois}

20 Le bois arrive au chantier soit déjà en planches, soit sous forme de billes qui seront débitées à la main par les scieurs de long. Leur travail consiste alors à découper, avec une précision absolue, un tronc d'arbre en fines planches de quelques millimètres. L'outil, qu'ils emploient en équipe de deux, est la scie à cadre, qui leur permet de travailler sur des troncs de gros diamètre (jusqu'à deux mètres).

21 Les planches qui composeront le bordé devront avoir une forme appropriée afin de suivre les lignes d'eau du bateau. Elles seront soumises à déformation par la chaleur, cintrées au feu. Leur cintrage est déterminé par leur emplacement (bordés du fond, pré-cintre...). La procédure, simple, est issue de la gestuelle immuable de la construction traditionnelle des bateaux du Vietnam.

Dès l'aube, après avoir posé sur un terre-plein la planche sur son chant, les ouvriers allument des feux de part et d'autre. La planche reste en position grâce à des poteaux auxquels elle est enchaînée. Deux heures s'écoulent, pendant lesquelles les ouvriers alimentent les foyers et veillent à ce que ni la planche ni les poteaux ne brûlent. Lorsque le bois est à bonne température, on commence à exercer une traction sur les extrémités de la planche afin d'obtenir une première déformation. Dans l'heure qui suit, on continue à tirer jusqu'à l'obtention de la courbe désirée.

23 Toujours enchaînée à ses poteaux, la planche est alors maintenue en tension jusqu'au lendemain par des écarteurs, pour que sa nouvelle forme soit conservée. Une trace noire, caractéristique indélébile de la méthode du cintrage au feu, persiste dans le creux du bordé.

24 Une fois cintrés, les bordés du fond sont fixés par des clous enfoncés en biais dans une encoche pré-coupée dans la planche; puis sont posées les varangues du fond et les membrures.

Les bordés qui composent le bordage supérieur, également ployés au feu, sont posés à franc bord à mesure que la construction avance. Fixés aux membrures et aux allonges, et maintenus en place au moyen de boulons, ils garantissent une meilleure tenue aux vibrations du moteur.

26 Les bordés ont une épaisseur de cinq à sept centimètres. Cela permet au bateau d'être chargé plus lourdement et de s'échouer ou de racler le fond sans problème. L'ossature 
transversale se termine avec la pose des allonges. A l'avant et à l'arrière, celles-ci se transforment en bittes d'amarrage, tru kéo dây. Celles qui débordent du livet, devenues inutiles, sont sciées une fois le bordé posé.

Lorsque la cale, long tàu, est terminée, on peut construire les superstructures, la cabine, phong ngủ, et le poste de pilotage, kiên.

\section{Le calfatage}

La pose des barrots du pont et des superstructures achève la construction. Il faut maintenant rendre la coque étanche grâce au calfatage, xảm tàu.

$\mathrm{Au}$ Vietnam, ce travail est réalisé par les femmes qui doivent acheter à la forge les outils nécessaires: un maillet, búa, trois fers à calfats, thúc (de trois, cinq et sept millimètres d'épaisseur), un fer à couper, xảm, une râpe, cao et un bec de corbeau, moc.

Le forgeron, thô rèn, fabrique tous les instruments nécessaires à la construction navale et au calfatage en fonction des besoins et de la morphologie de chacun, réalisant ainsi des outils très personnalisés.

31 Les matériaux de calfatage, tous d'origine naturelle, sont en revanche fournis par le chantier : la fibre de bambou, ba bô, la poudre de chai (chai bot) et l'huile de chai (dâu trong).

J'ai travaillé trois mois au sein d'une équipe de calfateuses qui m'ont enseigné tous les secrets de leur art : préparation des mélanges, pose du mastic et protection de la coque. Un bateau de trente mètres de long requiert trois kilomètres de calfatage ${ }^{9}$. Le nettoyage de la coque, le calfatage, la pose du mastic et des lisses en laiton sut les joints, ainsi que la peinture, sont effectués en trois semaines au plus.

Pour nous faciliter le travail, les ouvriers ont installé un « chemin aérien » le long de la ligne de flottaison. Suspendue dans le vide en position accroupie, le travail met à rude épreuve mon équilibre. À tour de rôle, nous préparons les mélanges, ce qui assure des repos de quelques heures à l'abri du soleil.

Le principe du calfatage est d'enfoncer avec énergie de l'étoupe séchée dans le joint qui se trouve entre deux bordés. Au contact de l'eau, cette fibre se dilate, colmate le joint et tend la coque étanche. L'art du calfatage réside dans le coup précis du maillet. L'étoupe entre dans le joint et vient occuper un espace dans lequel pas une seule goutte d'eau ne doit pénétrer.

Le calfatage commence par un bon équarrissage des joints des bordés, qui se fait avec un fer à calfat coupant. Ensuite, à l'aide d'un couteau en bambou on pose sur les deux faces du joint un mélange de poudre et d'huile de chai. Ce mélange glutineux et légèrement collant va recevoir la fibre de bambou et permettra de la retenir.

La fibre de bambou a été préparée à l'avance. Elle a été effilochée, libérée des éléments trop grossiers l'empêchant d'avoir une texture homogène. Puis elle a été imprégnée d'huile de chai, serrée sur elle-même et ensuite, effilochée de nouveau et aspergée avec de la poudre de chai afin que la matière soit légère et puisse pénétrer facilement dans la fissure du joint. Si l'étoupe déborde, on coupe le surplus avec le fer à calfat et, au moyen d'autres fers plus épais, on enfonce la matière, afin d'obtenir une ligne de calfatage homogène. La vie d'un bateau en bois dépend entièrement du calfatage. 
Après quoi, afin de rendre les joints parfaitement étanches et d'éviter ainsi la moisissure de l'étoupe, il faut poser un mastic.

\section{La protection de la coque}

Une fois la coque rendue étanche par le calfatage, on passe une couche de vernis naturel à base d'huile de chai ${ }^{10}$ pour la protéger avant la mise à l'eau.

L'avant du bateau est fortement soumis aux vagues, et l'arrière aux bouillonnements produits par les mouvements de l'hélice, quat vit. Dans ces deux parties, les joints doivent être renforcés avec des lisses en laiton clouées aux bordés du fond et rendues imperméables grâce à une couche de mastic répartie autour des bords. Cette technique est utilisée sur tous les bateaux maritimes. La coque des bateaux fluviaux est protégée par une épaisse couche de mastic recouverte de papier (on emploie des sacs de béton). Enfin, le tout est recouvert d'un film plastique et la coque est doublée avec des planches de bois très fines (cinq millimètres d'épaisseur, vingt centimètres de largueur sur sept mètres de longueur), clouées aux bordés du fond.

Les bateaux maritimes sont peints et leur coque est protégée par de la peinture antifouling ${ }^{11}$. Pour les préserver au mieux des tarets, con ha, il est conseillé de les caréner tous les ans ${ }^{12}$. Un bateau en bois naviguant en mer, caréné chaque année, a une durée moyenne de vie de 20 ans. Un bateau naviguant en rivière et ayant subi le même traitement a une durée de vie moyenne de 40 ans.

\section{L'âme d'un bateau}

41 Le bateau est un être vivant, et comme tel, possède une âme. Lorsqu'on est confronté à des phénomènes naturels impressionnants -orages, courants, rapides, tourbillons, marées, inondations-, on pense que tout cet univers qui nous dépasse est réglé par des forces surnaturelles. L'homme ne peut les dominer mais il sait, grâce à des rituels, les rendre bienfaisantes. Aux moments les plus importants de la vie d'un bateau, on sollicite donc l'aide des esprits ou des génies protecteurs. Ainsi, dans tous les chantiers navals, se trouve un autel consacré aux ancêtres, aux dieux protecteurs des marins, ou à la divinité d'un culte local ${ }^{13}$. Dans le chantier de Monsieur Ba Sai, à Nhà Bè, par exemple, se dresse l'autel de la pierre-génie, thân da, qui protège l'activité du chantier et les navires qui y sont fabriqués ${ }^{14}$.

$\mathrm{Au}$ moment de la pose de la structure axiale, on procède à une cérémonie (Cadière 1902 : 373). Au centre de la quille est déposé un plateau d'offrandes composées de fruits ${ }^{15}$, de trois verres d'alcool, d'un bouquet de fleurs, de papiers à brûler, de deux bougies et de trois bâtonnets d'encens. Un tissu écarlate en coton ou en soie, parfois accompagné de feuilles de cocotier (cây giua, Pandanus) est entouré et noué à l'extrémité de l'étrave. C'est un moment capital pour la réussite future du bateau. Cette cérémonie, suivie d'un festin, est menée à bien par le propriétaire et le constructeur.

Une nouvelle cérémonie interviendra lors de "l'ouverture des yeux » du bateau (le khai tâm khai quang), bien avant la fin de la construction, une fois la coque assemblée et avant le calfatage.

La phase d'ouverture des yeux débute très tôt le matin ( 5 h 30). À l'avant du bateau, mũi, on prend les mesures en vue de placer un pochoir qui délimite la forme d'yeux 
souhaitée, en veillant à ce qu'ils soient disposés à la même hauteur de chaque bord de la coque $^{16}$. On parfait le contour des yeux sur le bordé par une simple gravure effectuée à l'aide d'une pointe. Avant de continuer le travail, on procède à la cérémonie.

Le responsable du chantier ${ }^{17}$ a installé un autel devant le bateau. Sur un plateau, ont été disposées des offrandes : des fleurs, du raisin, des mangues, des papiers à brûler pour les ancêtres, deux bougies et six bâtonnets d'encens ${ }^{18}$, une assiette contenant du riz cru, du sel, trois bols vides ${ }^{19}$ et de l'argent. Le devin allume les trois bâtonnets d'encens, les deux bougies et adresse une prière aux ancêtres et aux génies protecteurs. Il termine cette première phase en versant du thé dans les trois bols.

Le propriétaire doit à son tour allumer l'encens, adresser sa prière aux ancêtres et aux génies protecteurs, verser du thé, brûler les papiers destinés aux ancêtres, jeter le riz et le sel à l'avant du bateau; il peut alors récupérer l'argent qui a été offert ${ }^{20}$. Les fleurs qui se trouvent sur l'autel sont ensuite déposées sur l'étrave ${ }^{21}$, à l'emplacement de l'autel propre au bateau. Les bols de thé vidés, l'encens, les bougies et le pochoir restent sur l'autel jusqu'à la fin du travail, qui reprend après la cérémonie.

La cérémonie d'ouverture des yeux est le moment symbolique où l'on donne une âme au bateau afin qu'il puisse naviguer en bonne et due forme. Un navire est une créature de la mer, comme l'est un poisson. Ses yeux lui permettront de percevoir les dangers procédant du fond des eaux et de les éviter. Le capitaine pense que les yeux peuvent le guider vers une bonne zone de pêche; mais ils permettront aussi de terroriser les requins qui confondront le bateau avec un poisson plus grand qu'eux. Les yeux sont également symbole de fortune et préviennent des maladies. Tous ceux et celles qui ont pris part à la construction sont invités à la cérémonie d'ouverture des yeux et les offrandes sont partagées entre les ouvriers présents. Puis on finit de sculpter les yeux à l'aide d'un couteau à bois. L'ouvrier fait en sorte d'obtenir un léger relief dans la pupille qui rend l'œil plus «vif». Lorsque le bateau n'est plus utilisé pour la navigation, ses yeux sont détachés de l'étrave et disposés sur un autel (photo 1).

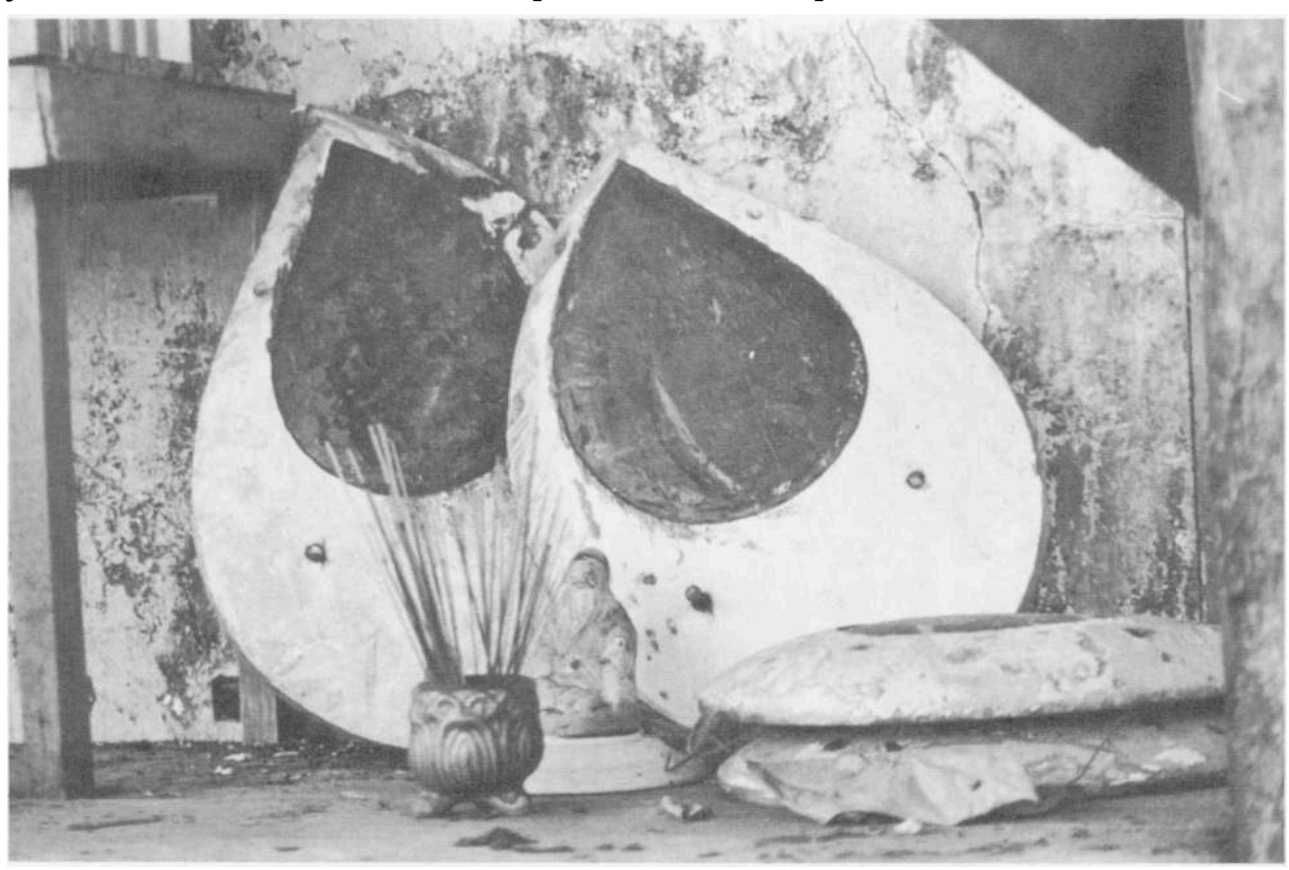

Photo 1. Les yeux d'un bateau hors d'usage disposées sur l'autel 
Seule la partie frontale des bateaux de transport du Delta du Mékong est décorée. Et l'on peint uniquement l'entrée de la cale et l'étrave, par où l'on accède à l'intérieur du bateau. Les couleurs employées sont le bleu, le rouge et le jaune. L'entrée de la cale est repeinte une fois par an, de préférence avant la fête du Têt, nouvel an vietnamien.

\section{Yeux, grecques, fleurs et signes des cartes}

Les caractéristiques des yeux varient selon le type de bateau et sa provenance. Sur les bateaux de Cho Lon et du Delta du Mékong, par exemple, les yeux sont peints en blanc et noir sur un fond rouge. Les ghe chài et les ghe cân đû̂ôc ont une étrave peinte en rouge et des yeux de grandes dimensions (environ $30 \mathrm{~cm}$ ). Sur les ghe cà vom (bateaux des provinces du nord et de l'est du Delta du Mékong), l'étrave et l'étambot sont peints en bleu et jaune avec des motifs floraux rappelant les décorations des temples chams, résultat de la superposition des influences chams, malaises et javanaises. Les yeux très petits $(10 \mathrm{~cm})$, sont placés sur la pièce d'étrave, qui ressemble à une trompe d'éléphant.

On trouve des grecques sur le ghe cà vom, et sur certains bateaux de la côte vietnamienne ${ }^{22}$.

Les fleurs à six pétales ont le même tracé que celles qui sont peintes sur la façade du Palais de la Cité Interdite de Pékin. D'origine chinoise, ce dessin a été reproduit sur les jonques et réutilisé par des propriétaires de bateaux chinois au Vietnam; il a ainsi marqué les mémoires.

Trèfle, coeur, pique et carreau remplacent de plus en plus l'ancre autrefois peinte sur l'étrave de bateaux de transport. Les Vietnamiens, en effet, aiment beaucoup les jeux de cartes; ils jouent souvent à bord des bateaux, lors des navigations, et ce n'est donc pas un hasard si ces symboles ont été choisis. Par ailleurs, il est certain que l'influence de la symbolique chinoise ${ }^{23}$ est particulièrement forte. De longue date, les Chinois installés au Vietnam sont des gens de négoce qui possèdent ou affrètent les bateaux de transport.

Le symbole de la dualité yin et yang est présent dans les ghe cà vom, en particulier sur le gouvernail, là où les efforts et les forces doivent se rencontrer et s'équilibrer. On le trouve peint en noir et blanc, en blanc et jaune, ou encore en rouge et jaune.

Somme toute, l'étude menée sur l'activité d'un chantier naval à Hochiminh-Ville, où l'on construisait encore, selon la tradition, des bateaux en bois, m'a permis de connaître les trois types représentatifs de la batellerie du Vietnam. Les trois embarcations du Mékong que j'ai décrites sont le ghe chài, le ghe cân đûôc, le ghe cà vom. Leurs caractéristiques peuvent être modifiées selon les besoins du propriétaire. Mais la décoration et les formes sont maintenues; cela permet de les différencier et de reconnaître l'origine du bateau.

Aujourd'hui, dans la majorité des chantiers, on construit des bateaux en métal et les formes changent. Des bateaux du Mékong, ne restent que de vieilles unités, dont les « yeux » caressent le flot qui coule vers la mer. 


\section{BIBLIOGRAPHIE}

Audemard, L., 1970, Les jonques chinoises, IX : côtes est. Rotterdam : Museum voor land and Volkenkunde en het Maritiem Museum « Prius Hendrick ».

Cadière, Leopold

- 1902, «Cérémonie pour la construction d'une jonque », Bulletin de l'École Française d'Extrême Orient (BEFEO) : 373-376.

Gage, William W., 1976, « Probing the history of Vietnamese tones ", in Actes du XXIXe Congrès international des Orientalistes, Asie du Sud-Est continentale (vol. 1). Paris : L’Asiathèque.

Paris, Pierre, 1942, «Esquisse d'une ethnologie navale des pays annamites », Bulletin des Amis du Vieux Hué 4.

\section{NOTES}

2. Arroyo : ce mot espagnol désigne souvent des rivières peu importantes.

3. Can Duoc se trouve à environ $30 \mathrm{~km}$ au sud de Hochiminh-Ville.

4. En 1995, toute activité a cessé en raison d'un problème de corruption. Une partie des affaires a été reprise par le chantier voisin, celui de M. Ba Sai.

5. Au Vietnam, il ne faut pas hésiter à commencer la construction d'un bateau pendant les jours qui portent les numéros 9,19 et 29 ; il faut en revanche éviter formellement les jours 7,17, 27.

6. Mũi en vietnamien signifie nez et sert à désigner l'étrave d'un bateau. C'est un nom d'origine Mon-Khmèr (Gage 1976, vol. $1: 61$ ).

7. Ce mode d'assemblage est encore pratiqué sur la plage de Mui Ne, dans la province de Phan Thiet; sur l'île Cam à Hoi An et sur la plage de Dong Hoi dans la province du Quang Binh.

8. En architecture navale, lorsqu'on dessine un bateau, on fait un plan de ses lignes d'eau qui permet de lire sa forme et ses caractéristiques nautiques.

9. La rémunération de la main-d'œuvre pour le calfatage est de mille deux cents dôngs (cinquante centimes) par mètre linéaire et comprend le nettoyage du joint, le nouveau calfatage et la pose du mastic.

10. Au contact de l'eau, ce vernis devient blanc.

11. La peinture antifouling est à base de cuivre : cela empêche le développement de parasites sur la coque.

12. J'ai observé que, de plus en plus souvent, les œuvres vives des bateaux sont protégées par une fine couche de béton qui reste en place grâce à une trame de fil barbelé fixée à la coque en bois.

13. Le panthéon vietnamien est un mélange de bouddhisme Mahayana (grand véhicule), de taoïsme, de confucianisme et de culte des ancêtres. Les génies occupent une place importante dans les activités humaines et sont présents et célébrés lors de la récolte, lors des navigations et des rituels liés à la construction en général.

14. Pendant la période de l'exode vietnamien, le chantier a construit des bateaux pour les boatpeople, qui tous sont arrivés sans encombre à destination. Aujourd'hui encore, les réfugiés retournés au Vietnam se rendent au chantier pour remercier le propriétaire et faire des offrandes à la pierre-génie.

15. Pomme, cannelle, raisin, mangue, fruit-dragon, longane.

16. Quand on regarde le bateau dans son axe central, les yeux ont toujours tendance à loucher. 
17. C'est souvent le constructeur ou le chef du chantier qui joue le rôle du devin lors des cérémonies.

18. Trois bâtonnets d'encens seront utilisés par le devin et trois par le propriétaire du bateau.

19. Pour les trois Joyaux: Dharma, Sangha, Bouddha, qui représentent à la fois l'avenir, le présent et le passé.

20. Cette offrande est de l'ordre de 50000 dongs (30 F).

21. Il faut éviter de stationner trop longtemps dans cette partie du bateau et de toucher ou maltraiter l'étrave, car les esprits y ont leur demeure.

22. On trouve cette décoration sur les bateaux de pêche de la plage de Mui Ne dans la province de Phan Thiet, le long de la côte, à $140 \mathrm{~km}$ au nord de Hochiminh-Ville.

23. Le tao est le symbole le plus répandu dans la décoration des jonques du Fou Kien et du Tché Kiang (côte est de la Chine). Cf. Audemard (1970:12).

\section{RÉSUMÉS}

Cet article s'intéresse à trois types de bateaux au Vietnam qui naviguaient sur les fleuves et les eaux du Delta et servaient principalement au transport. Leur description, associée à L'étude d'un chantier naval, permet de dégager les constantes et les innovations concernant les bateaux en bois. La description de la filière économique et technique menant à la réalisation d'un bateau permet de comprendre le rôle des différents acteurs impliqués (propriétaires, architectes, ouvriers et parmi eux le rôle des femmes) et situe dans son contexte technique les expressions rituelles propres aux bateaux du Vietnam.

This article takes interest with three types of boat in Vietnam, which sailed on the waters of the rivers and the delta, as means of transport. The description linked with the study of a naval building site lead to draw the persistences and the innovations concerning wooden boats. Through the description of the different stages - economical and technical - to the achievement of the boat, we can understand the role of all the actors implied (owners, architects, workers, and also women) and place in the technical context, the rituals expressions associated to the construction of Vietnamese boats.

Este artículo concierne a tres tipos de embarcaciones del Vietnam que navegaban por las aguas de los ríos y las del delta y servían principalmente para el transporte. Su descripción, asociada al estudio de un astillero, permite destacar las constantes y las ¡novaciones en lo que atañe a la carpintería de rivera. La descripción de la sucesión de medios económicos y técnicos que conduce a la realización de un barco, permite comprender el papel de cada uno de los diferentes actores implicados (propietarios, arquitectos, obreros y entre ellos el papel de las mujeres). También coloca en su contexto técnico las manifestaciones rituales inherentes a las embarcaciones vietnamitas.

\section{INDEX}

Mots-clés : chantiers navals, décoration, Delta du Mékong, rituels, Vietnam 
AUTEUR

LAURA BOGANI

Doctorante, Techniques et culture, 27 rue Paul Bert, 94204 Ivry Cedex 